\title{
Interionic Potentials for Some Alkali Halides from Crystal Data Measured at Different Temperatures
}

\author{
Silvano Romano, Chiara Margheritis, and Cesare Sinistri \\ Centro di studio per la termodnamica ed elettrochimica dei sistemi salini fusi e solidi del CNR \\ (Pavia, Italy), Institute of Physical Chemistry, University of Pavia, Italy
}

(Z. Naturforsch. 29a, 1202-1205 [1974] ; received May 15, 1974)

\begin{abstract}
Values at different temperatures of lattice constants and their derivatives with respect to $T$. and of elastic constants were used to obtain the derivatives with respect to the minimum interionic distance of the repulsive potentials for the crystals $\mathrm{CsCl}, \mathrm{CsBr}, \mathrm{ClI}, \mathrm{NaCl}, \mathrm{KCl}$ and $\mathrm{KBr}$. The derivatives thus calculated were then subjected to a computer fitting to yield the $a_{i j}$ and $b$ constants of the interionic repulsive pair potential: $\mathrm{R}_{\varphi i j}=a_{i j} \exp \left\{-b r_{i j}\right\}$.
\end{abstract}

Interionic potentials are of present interest for the general prediction of thermodynamic properties $^{1}$ and for computer simulation by Monte Carlo or molecular dynamics methods ${ }^{2}$.

Fumi-Tosi in $1964^{3}$ reported interionic potentials for NaCl-type alkali halides which represent a successful attempt to characterize this family of salts. These authors, however, were particularly concerned with the problem of ionic sizes: they assumed the Huggins-Mayer model ${ }^{4}$ for the Born repulsive energy and used input data at only one temperature, together with Mayer's constants which have been later criticized ${ }^{5}$.

To obtain the pair potentials of a single salt, it seems more appropriate to fit only data of that salt in a way as much independent from models as possible.

For some solid alkali halides, remarkably precise crystal data in a wide temperature range are now available. These data, in a temperature range limited to $300-700{ }^{\circ} \mathrm{K}$ in order to neglect anharmonic contributions, were used in the present work to obtain interionic potentials for the three caesium halides $\mathrm{CsCl}, \mathrm{CsBr}$, CsI and for $\mathrm{NaCl}, \mathrm{KCl}, \mathrm{KBr}$.

\section{Calculations}

The average energy $\Phi$ of an ion in the perfect crystal lattice is taken as formed by a coulombic, a van der Waals and a repulsive term:

$$
\Phi(\boldsymbol{r})={ }^{\mathrm{C}} \Phi(\boldsymbol{r})+{ }^{\mathrm{W}} \Phi(\boldsymbol{r})+{ }^{\mathrm{R}} \Phi(\boldsymbol{r}) .
$$

$\Phi$ is only a function of the smallest interionic distance $\boldsymbol{r}$ since all of the interionic distances can be related to $\boldsymbol{r}$ by geometrical constants. In fact, the relation between $\Phi$ and the pair potentials $\varphi_{i j}$ for the ions $i$ and $j$ up to the $5^{\text {th }}$ co-ordination sphere can be written:

for a body-centred cubic arrangement (b.c.c.)

$$
\begin{aligned}
\Phi=8 \varphi_{\mathrm{u}}(\boldsymbol{r})+ & \frac{6}{2} \varphi_{1}\left(\sqrt{\frac{4}{3}} \boldsymbol{r}\right)+\frac{12}{2} \varphi_{1}\left(\sqrt{\frac{\overline{8}}{3}} \boldsymbol{r}\right) \\
& +24 \varphi_{\mathrm{u}}\left(\sqrt{\frac{11}{3}} \boldsymbol{r}\right)+\frac{8}{2} \varphi_{1}(2 \boldsymbol{r})
\end{aligned}
$$

for a body-centred cubic arrangement (b.c.c.):

$$
\begin{array}{r}
\Phi=6 \varphi_{\mathrm{u}}(\boldsymbol{r})+\frac{12}{2} \varphi_{1}(\sqrt{2} \boldsymbol{r})+8 \varphi_{\mathrm{u}}(\sqrt{ } 3 \boldsymbol{r}) \\
+\frac{6}{2} \varphi_{1}(2 \boldsymbol{r})+24 \varphi_{\mathrm{u}}(\sqrt{5} \boldsymbol{r})
\end{array}
$$

with $\varphi_{1} \equiv \varphi_{++}+\varphi_{\ldots}$. and $\varphi_{\mathrm{u}} \equiv \varphi_{+} \ldots$

The Hildebrand equation of state

$$
N(\partial \Phi / \partial V)_{T}=T \alpha / \beta_{\text {is }}-P,
$$

written in the form

$$
(\partial \Phi / \partial \boldsymbol{r})_{T}=k \boldsymbol{r}^{2}\left(T \alpha / \beta_{\text {is }}-P\right) 10^{-24}
$$

was used for the calculations, where $\alpha=$ thermal volume expansion coefficient, $\beta_{\text {is }}=$ isothermal compressibility, $k=$ structural constant, equal to 4.6188 for a b.c.c. structure and to 6 for a f.c.c. structure; $\boldsymbol{r}$ in $\AA$.

Equation (5) allows to obtain the experimental values of $(\partial \Phi / \partial \boldsymbol{r})_{\mathrm{T}}$ if the lattice constants and their derivatives with respect to $T$ (to evaluate $\alpha$ ) and the elastic adiabatic constants and $C_{\mathrm{P}}(T)$ data (to evaluate $\beta_{\text {is }}$ ) are at disposal. Then, with Eq. (1), values of $\left(\partial^{\mathrm{R}} \Phi / \partial \boldsymbol{r}\right)_{T} \equiv{ }^{\mathrm{R}} \Phi^{\prime}$ can be calculated at each temperature provided that ${ }^{\mathrm{C}} \Phi(\boldsymbol{r})$ and ${ }^{\mathrm{W}} \Phi(\boldsymbol{r})$ are known.

The coulombic term is well known. The term ${ }^{\mathrm{W}} \Phi$ has been taken as ${ }^{\mathrm{W}} \Phi=-C r^{-6}-D r^{-8}, C$ and $D$ being the van der Waals dipole-dipole and dipolequadrupole coefficients. These coefficients have been evaluated first by Mayer ${ }^{6}$ in 1933 and later by Hajj ${ }^{7}$. For a small number of alkali halides they have been critically reviewed by Lynch ${ }^{5}$. In the 
following calculations the $C$ values were taken from Lynch when available or else from Hajj. The $r^{-8}$ term was neglected. For a comparison with the F. T. results, calculations were also carried out with the original $C$ and $D$ Mayer's constants.

The experimental values of ${ }^{\mathrm{R}} \Phi^{\prime}$ at different temperatures were used to obtain the repulsive pair potentials between the ions $i$ and $j$ for each salt. For these potentials the exponential dependence on the interionic distance $r_{i j}$ :

$$
{ }^{\mathrm{R}} \varphi_{i j}=a_{i j} \exp \left\{-b r_{i j}\right\}
$$

was assumed, where $a_{i j}$ and $b$ are characteristic constants.

In the calculations a particular value of $b$ was chosen: by a least squares computer fitting procedure it was then possible to obtain values of $a_{1}=a_{++}+a_{--}$and $a_{\mathrm{u}}=a_{+-}$which reproduce the ${ }^{\mathrm{R}} \Phi^{\prime}$ data over the whole temperature range. Thus a group of three constants $b, a_{1}, a_{\mathrm{u}}$ characterized by a value $s^{\prime}$ of the standard deviation was obtained: by varying $b$ in suitable intervals the function $s^{\prime}(b)$ shows a minimum which singles out the best group of $b, a_{1}, a_{\mathrm{u}}$.

To complete this type of analysis, from the experimental values of ${ }^{\mathrm{R}} \Phi^{\prime}$ the values of the second derivatives ${ }^{\mathrm{R}} \Phi^{\prime \prime}$ and the increments $\Delta^{\mathrm{R}} \Phi$ of the repulsive potentials were also calculated. These quantities were then fitted in a similar way using the groups $b, a_{1}, a_{\mathrm{u}}$ previously obtained: in general the minima of the standard deviations as a function of $b$ agree very well. Since the function ${ }^{\mathrm{R}} \Phi^{\prime \prime}$ is the most sensitive to the $b$ values, the group of constants corresponding to the minimum of the $s^{\prime \prime}(b)$ was preferred. As an example, the minima obtained for $\mathrm{C}_{3} \mathrm{Cl}$, using Hajj's constant to evaluate ${ }^{\mathrm{W}} \Phi$, are at $b=2.53$ for $s(b)$, at $b=2.48$ for $s^{\prime}(b)$, and at $b=2.54$ for $s^{\prime \prime}(b)$.

Table 1 reports the chosen values of $b, a_{1}, a_{n}$ for the examined salts: in the first column there are the data obtained with Lynch's or Hajj's van der Waals coefficients, in the second one the data obtained with Mayer's constants, and in the third one, for comparison, F. T.'s data.

Figures 1 and 2 report the groups of $b, a_{1}, a_{u}$ constants obtained for the studied salts using Lynch's or Hajj's data. The minima of the $s^{\prime \prime}(b)$ function are indicated by an arrow. It is apparent, particularly for the caesium halides, that the range of $b$ in which $a_{1}$ and $a_{\mathrm{u}}$ are physically significant is very narrow.
Table 1. Interionic potential parameters for some alkali halides. The data were obtained using for $W \Phi$ Lynch's or Hajj's constants (column I), Mayer's constants (column II). Fumi-Tosi's values are in column III. $b$ is in $\AA^{-1}, a_{u}$ and $a_{l}$ in erg/molecule *.

\begin{tabular}{|c|c|c|c|c|}
\hline Salt & & I & II & III \\
\hline $\mathrm{CsCl}$ & $\begin{array}{l}a_{l} \\
b\end{array}$ & $\begin{array}{l}0.846 \cdot 10^{-8} \\
0.110 \cdot 10^{-8} \\
\quad 2.54\end{array}$ & $\begin{array}{l}0.121 \cdot 10^{-7} \\
0.134 \cdot 10^{-8} \\
\quad 2.65\end{array}$ & \\
\hline $\mathrm{CsBr}$ & $\begin{array}{l}a_{l} \\
a_{u} \\
b\end{array}$ & $\begin{array}{l}0.590 \cdot 10^{-8} \\
0.881 \cdot 10^{-9} \\
\quad 2.35\end{array}$ & $\begin{array}{l}0.895 \cdot 10^{-8} \\
0.117 \cdot 10^{-8} \\
\quad 2.49\end{array}$ & \\
\hline CsI & $\begin{array}{l}a_{l} \\
a_{u} \\
b\end{array}$ & $\begin{array}{l}0.138 \cdot 10^{-7} \\
0.194 \cdot 10^{-8} \\
\quad 2.42\end{array}$ & $\begin{array}{l}0.302 \cdot 10^{-7} \\
0.360 \cdot 10^{-8} \\
\quad 2.66\end{array}$ & \\
\hline $\mathrm{NaCl}$ & $\begin{array}{l}a_{l} \\
a_{u} \\
b\end{array}$ & $\begin{array}{l}0.438 \cdot 10^{-8} \\
0.417 \cdot 10^{-9} \\
\quad 2.72\end{array}$ & $\begin{array}{l}0.432 \cdot 10^{-8} \\
0.419 \cdot 10^{-9} \\
\quad 2.70\end{array}$ & $\begin{array}{l}0.626 \cdot 10^{-8} \\
0.201 \cdot 10^{-8} \\
\quad 3.15\end{array}$ \\
\hline $\mathrm{KCl}$ & $\begin{array}{l}a_{l} \\
a_{u} \\
b\end{array}$ & $\begin{array}{l}0.105 \cdot 10^{-7} \\
0.765 \cdot 10^{-9} \\
\quad 2.64\end{array}$ & $\begin{array}{l}0.121 \cdot 10^{-7} \\
0.830 \cdot 10^{-9} \\
\quad 2.68\end{array}$ & $\begin{array}{l}0.558 \cdot 10^{-8} \\
0.286 \cdot 10^{-8} \\
\quad 2.97\end{array}$ \\
\hline $\mathrm{KBr}$ & $\begin{array}{l}a_{l} \\
a_{u} \\
b\end{array}$ & $\begin{array}{l}0.144 \cdot 10^{-7} \\
0.910 \cdot 10^{-9} \\
\quad 2.60\end{array}$ & $\begin{array}{l}0.171 \cdot 10^{-7} \\
0.102 \cdot 10^{-8} \\
2.65\end{array}$ & $\begin{array}{l}0.976 \cdot 10^{-8} \\
0.447 \cdot 10^{-8} \\
2.99\end{array}$ \\
\hline
\end{tabular}

In Figure 2 the values obtained by F. T. are also indicated. These values, particularly $a_{\mathrm{u}}$, are consistent with the reported functions even though the $b$ values are always larger than those obtained by the minimum of the standard deviation. This might be due to the fact that F. T. fitted at a single temperature data of the whole alkali halide family, while, in the present work, the fitting was done salt by salt on data at different temperatures.

It should still be noted that the values of the repulsive energy $N^{\mathrm{R}} \Phi$ that can be calculated by the groups of $b, a_{1}, a_{u}$ reported in Figs. 1, 2 are very close for relatively wide intervals of $b$. For example,

\footnotetext{
* The input data were, at different temperatures: a) values of lattice constant and its derivative in respect to $T$ reported by P. D. Patak and N. G. Vasavada (Acta Cryst. A 26, 655 [1970]) for $\mathrm{NaCl}, \mathrm{KCl}$ and $\mathrm{CsBr}$; by P. D. Patak and N. V. Pandya (Indian J. Phys. 34, 416 [1960]) for $\mathrm{KBr}$; by J. W. Menary, A. R. Ubbelohde, and I. Wood. ward (Proc. Roy. Soc. London A 208, 158 [1951]) for $\mathrm{CsCl}$ and by J. W. Johnson, P. A. Agron, and M. A. Bredig (J. Amer. Chem. Soc. 77, 2734 [1955]) for CsI; b) values of elastic constants reported by O. D. Slagle and H. A. Mc Kinstry (J. Appl. Phys. 38, 437 and 451 [1967]) for all the salts; c) values of $C_{\mathrm{p}}$ reported by $\mathrm{A}$. J. Leadbetter and G. R. Settatree (J. Phys. C (Solid St. Phys.) 2, 385 [1969]) for $\mathrm{NaCl}, \mathrm{KCl}$ and $\mathrm{KBr}$ and by M. Blander ("Molten Salt Chemistry", J. Wiley Ed., New York 1964, pag. 141) for caesium halides.
} 

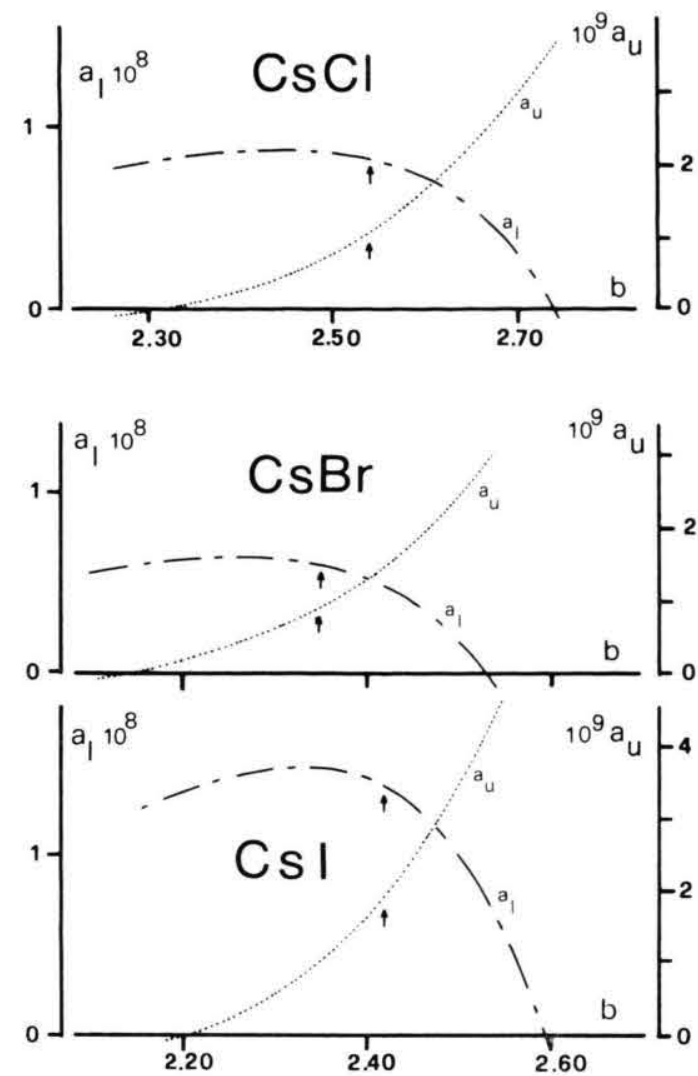

Fig. 1. Values of $a_{1}$ and $a_{u}$ as functions of $b$ for the three caesium halides. The arrows show the data corresponding to the minimum of the standard deviation function of the second derivative of the repulsive energy.

in the case of $\mathrm{CsCl}$ at $303{ }^{\circ} \mathrm{K}$ the following values in $\mathrm{kcal} / \mathrm{mole}$ are obtained: 25.3 at $b=2.33 ; 25.4$ at $b=2.54$ and 25.2 at $b=2.73$. Thus, values of $N^{\mathrm{R}} \Phi$ calculated with F.T. data are in satisfactory agreement with the present results.

As regards the precision of the constants $b, a_{1}, a_{\mathrm{u}}$ it was proved that a variation of $\pm 1 \%$ in $\alpha$ and $\mp 2 \%$ in $\beta_{\text {is }}$ in Eq. (5) (quoted errors for the input experimental data) leads to an uncertainty of about $\pm 2 \%$ in $b, \pm 15-20 \%$ in $a_{1}, \pm 10-15 \%$ in $a_{\mathrm{u}}$ and $\mp 2 \%$ in the total repulsive energy.

As a final remark, it can be observed that assuming the following inverse power form for the repulsive pair potential:

$$
\mathrm{R}_{\varphi_{i j}}=a_{i j} r_{i j}^{-b}
$$

the values of ${ }^{\mathrm{R}} \Phi^{\prime}$ and ${ }^{\mathrm{R}} \Phi^{\prime \prime}$ are given by

$$
{ }^{\mathrm{R}} \Phi^{\prime}=-b \boldsymbol{r}^{-b-1}\left(f_{\mathrm{u}} a_{\mathrm{u}}+f_{1} a_{1}\right),
$$

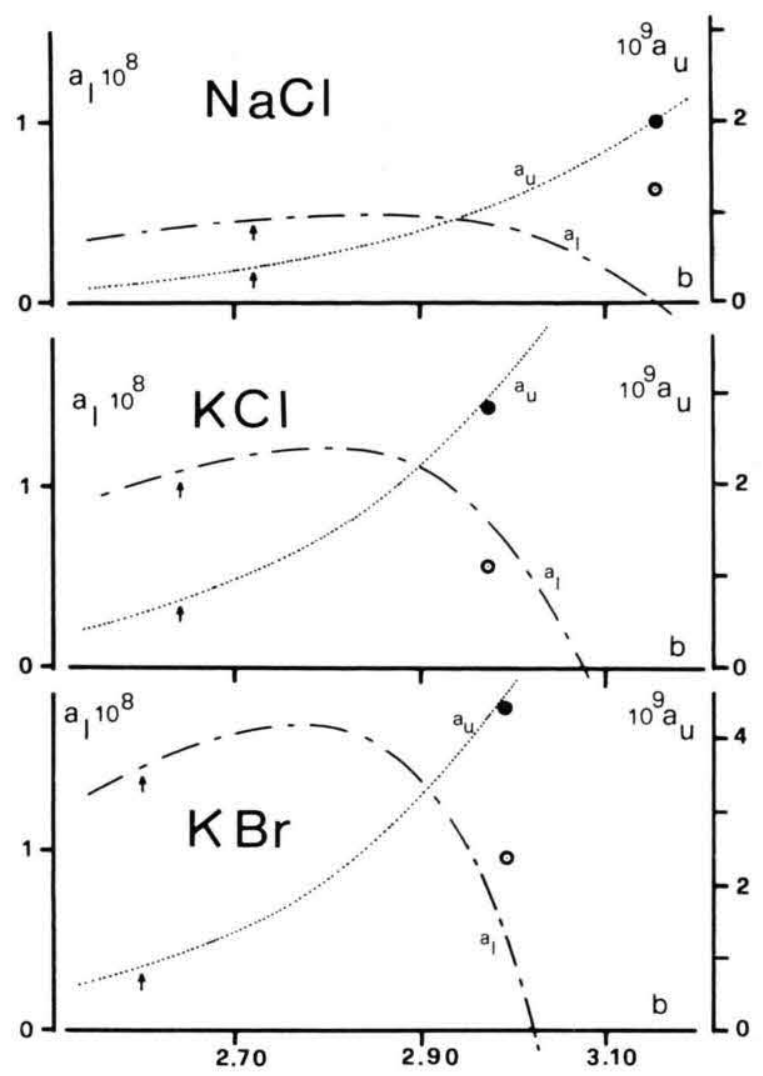

Fig. 2. Values of $a_{1}$ and $a_{\mathrm{u}}$ functions of $b$ for $\mathrm{NaCl}, \mathrm{KCl}$ and $\mathrm{KBr}$. The arrows show the data corresponding to the minimum of the standard deviation function of the second derivative of the repulsive energy. Values of F. T. ${ }^{3}$ are indicated with small circles ( $\bigcirc$ for $a_{1}$ and for $a_{\mathrm{u}}$ ).

$$
{ }^{\mathrm{R}} \Phi^{\prime \prime}=b(b+1) \boldsymbol{r}^{-b-2}\left(f_{\mathrm{u}} a_{\mathrm{u}}+f_{1} a_{1}\right)
$$

where $f_{\mathrm{u}}$ and $f_{1}$ are geometrical constants. From Eqs. $(8,9)$ it follows:

$$
b=-\boldsymbol{r}\left({ }^{\mathrm{R}} \Phi^{\prime \prime} / \mathrm{R} \Phi^{\prime}\right)-1 .
$$

Evaluation of $b$ by means of Eq. (10) was thought to be a sensitive test for the applicability of relation (7) to the crystals studied in the present work. It was generally found that $b$ is not a constant: it depends on temperature and thus on $\boldsymbol{r}$; e. g. going from $303^{\circ} \mathrm{K}$ to $663^{\circ} \mathrm{K} b$ varies from 9.73 to 7.47 for $\mathrm{CsCl}$ and from 9.29 to 7.95 for $\mathrm{KCl}$.

\section{Acknowledgement}

We are indebted to Prof. M. P. Tosi (MessinaItaly) for helpful discussions and suggestions. 
1 F. H. Ree and A. C. Holt, Phys. Rev. B 8, 826 [1973].

${ }^{2}$ see e.g.: L. V. Woodcock and K. Singer, Trans. Faraday Soc. 67, 12 [1971] ; S. Romano and I. R. McDonald, Physica 67, 625 [1973].

${ }^{3}$ F. G. Fumi and M. P. Tosi, J. Phys. Chem. Solids 25, 31 and 45 [1964].
4 M. L. Huggins and J. E. Mayer, J. Chem. Phys. 1, 643 [1933]; M. L. Huggins, J. Chem. Phys. 5, 143 [1937], ibid. 15, 212 [1947].

5 D. W. Lynch, J. Phys. Chem. Solids 28, 1941 [1967].

6 J. E. Mayer, J. Chem. Phys. 1, 270 [1933].

7 F. Hajj, J. Chem. Phys. 44, 4618 [1966]. 